\title{
Asymptotische Entwicklungen der Gaußschen hypergeometrischén Funktion für unbeschränkte Parameter
}

\author{
E. WAGNEI
}

Meinem verehrten Lehrer Prof. Dr. L. Berg in Dankbarkeit anlüßlich seines 60. Geburtstages gewidmet

Für die hypergeometrische Funktion $F(a, b ; c ; z)$ werden asymptotische Entwịcklungè im Fall $a^{2}=o(c), b^{2}=o(c)(|c| \rightarrow \infty)$ hergeleitet.

Выноднтся асимптотические разлокения гипергеометрической фу!нции $F(a, b ; c ; z)$ в случас $a^{2}=o(c), b^{2}=o(c)(|c| \rightarrow \infty)$.

$$
\text { i. }
$$

A'symptotic expansions of the hypergeometric function $F(a, b ; c ; z)$ are derived in the case $\cdot a^{2}=o(c), b^{2}=o(c)(|c| \rightarrow \infty)$.

\section{Finleitung}

In [9] 'werden asymptotische Entwicklungen der Gaußschen hypergeometrischen Funktion $F(a, b ; c ; z)$ fïr beliebig gegen Unendlich strebende komplexe Werte von $c(c \neq 0,-1, \ldots)$ bei konstanten Werten von $a, b, z(|\arg (1-z)| \leqq \pi)$ hergeleitet und damit die in [1] aufgestellte Behauptung widerlegt, daß die hypergeometrische Reihe in allen Punkten ihres Konvergenzgebiets $(|z|<1)$. gleichzeitig asymptotische Entwicklung vón $F$ für $|c| \rightarrow \infty$ ist. In der vorliegenden Arbeit wird der Gültigkeitsbereich dieser Entwicklungen auf unbeschränkte Parameter $a, b$ unter den. generellen Voraussetzungen $a^{2}=o(c), b^{2}=o(c)$ sowie gewissen Beschränktheitsbedingungen für die Realteile von, $a$ und $b$ ausgedehnt. Obwohl dảbei vón der gleichen Beweiskonzeption, wie in [9] ausgegangen wird, erfordert die Nichtanwendbarkeit des Lemmas von Watson eine andere Methodik, die sich wesentlich auf allgemeine Prinzipien und Sät:ze der Monogràphie [2] von' Berg stiitzt.

Asymptotische Entwicklungen von $F(a, b ; c ; z)$ für mehrere gegen Unendlich strebende Parameter sind bisher nur in speziellen Fällen bekannt, in denen die unbeschränliten_Parameter durch Nebenbedingungen miteinander verkniipft sind (siehe. z. B. [4] mit $u={ }^{\prime} \alpha l, c=\gamma l$ für $l \rightarrow \infty$ und konstante $\alpha, b, \gamma$ oder [5, 11], wo die Differenzen oder Summen der unbeschränkten Parameter konstant sind). In. [7] werden asymptotische Darstellungen fiir $|a| \rightarrow \infty, b^{2}=o(a) ; c^{2}=o(a)$ 'und'einseitig beschränkte Realteile von $a$ und. $b$ angegeben.

Angeregt wurden die'Untersuchungen in dieser und den vorangehénden Arbeiten [6-9] des-Verfassers durch das Auftreten der Funktion $F$ bei der mathematischen Behandlung gewisser Polymerisationsprozesse, wobei die 'Parameter als Ketten'längen von Makromolekïlen in Erscheinung treten und $F$. wesentlich die Molmassenverteilung im Polymerisat bestimmt: 


\section{Bezeichnungen und Resultate}

Im folgenden verwenden wir die Bezeichnungen $a_{:}=\operatorname{Re} \cdot a, a_{2}=\operatorname{Im} a, \alpha=\operatorname{Arg} a$ $\therefore(-\pi, \pi]$ (entsprechend $\left.b_{1}, b_{2}, \beta, c_{1}, c_{2}, \gamma\right), x=\operatorname{Re} z, y=\operatorname{Im} z, \zeta=\operatorname{Arg} z, \log z$. $=\ln |z|+i \zeta$ und das Pochhammer-Symbol.

$$
(a)_{n}=\frac{\Gamma(u+n)}{-\Gamma(a)}= \begin{cases}1 & \text { für } n=0, \\ a(a+1) \ldots(a+n-1) . & \text { für } n=1,2, \ldots\end{cases}
$$

$\therefore$ Für jedes $z$, mit $x>1 / 2 ; z \notin[1 ; \infty)$ setzen wir

$$
\Phi_{z}^{(k)}=\operatorname{Arg}(-\log (1-1 / z)+2 k \pi \mathrm{i}) \quad(k=-1,0,1) .
$$

Die für jedes' $\zeta \in(0, \pi / 2)$ echt monoton wachsende Funlition

$$
g ;(\Phi)=\ln [\sin \Phi / \sin \zeta]-(\pi+\Phi, \zeta) \cot \Phi \quad\left(\zeta<\Phi^{\prime}<\pi-\zeta\right)
$$

besitzt genau cine. Nullstelle in $(\zeta, \pi / 2)$, die wir mit. $\Phi_{*}^{*}$ bezeichnen. Dann sei für $y \geqq 0$.

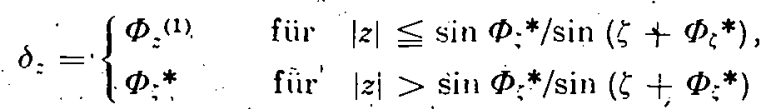

und fiir $\dot{y}<0$

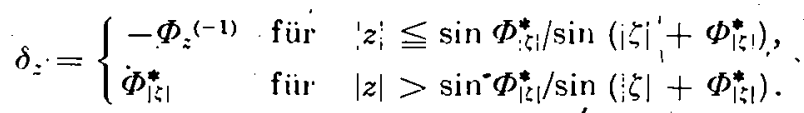

Offenbar sind $\Phi_{z}{ }^{(0)} \in(-\pi / 2,0)$ für $y \geqq 0, \Phi_{z}{ }^{(0)} \in(0, \pi / 2)$ für $y<0$ sowie $\delta_{z} \in(0$, $\pi / 2)$ und $0<\sin \Phi_{i}^{*} / \sin \left(\xi+\Phi_{\zeta}^{*}\right)$.

Unter $\varepsilon$ ist stets eine beliebig klein wählbare positive Konstante zu verstehen. Nach - unten besçränkte reelle Variable werden durch $O_{L}(1)$ gekennzeichnet. Die Ergebnisse der vorliegenden Arbeit sind in folgenden Satz zusammengefaßt:

-. Satz: Essei

$$
\begin{array}{ll}
z \neq 0, \ldots & z \notin[1, \infty), \quad \\
a^{2}=o(c), & b^{2}=o(c) \\
a_{1}=O_{L}(1), & b_{1}=O_{L}(1) .
\end{array}
$$

Dann gilt in jedem der vier Fülle.

$$
a=0,-1,-2, \ldots \text { oder } b=0,-1,-\frac{1}{2}, \ldots,
$$

(iii) $\quad x>1 / 2, y \geqq 0,-\pi / 2-\delta_{z}+\varepsilon \leqq \gamma \leqq \pi / 2-\Phi_{z}{ }^{(0)}-\varepsilon$,

(iv) $\quad x>1 / 2, y<0,-\pi / 2-\Phi_{z}{ }^{(0)}+\varepsilon \leqq \gamma \leqq \pi / 2+\delta_{z}-\varepsilon$

- die asymptotische Entwicklung

$$
F(a ; b ; c ; z) \approx \sum_{v=0}^{\infty} \frac{(a)_{\nu}(b)_{v}}{v^{\prime} !(c)_{\nu}} z^{v} \quad(c \rightarrow \infty)
$$

Sind $a, b \neq 0,-1,-2, \ldots$ und stalt $(6)$ die schärferen Bedingungen.

$$
u_{1}=O(1), \quad b_{1} \doteq O(1)
$$


erfüllt, so gelten in jedem der zuci Fialle .

(v) $\quad x>1 / 2, y \geqq 0,-\pi+\varepsilon \leqq \gamma \leqq-\pi / 2-\Phi_{i}^{(1)}-\varepsilon$ und $\pi / 2-\Phi_{z}{ }^{(0)}+\varepsilon$ $\leqq \gamma \leqq \pi-\dot{\varepsilon}$,

(vi)

$$
x>:_{1} 1 / 2, \cdot y<0,-\pi+\varepsilon \leqq y \leqq-\pi / 2-\Phi_{z}{ }^{(0)}-\varepsilon \text { und } \pi / 2-\Phi_{z}^{(-1)}+\dot{\varepsilon}
$$

1. $\leqq \gamma \leqq \tau-\varepsilon$

gleichzeitig die beiden asymptotischen Entuicklungen

$F(a, b ; c ; z) \approx \frac{\pi \Gamma(a-c+1) \Gamma(b-c+1) z^{1-c}(1-z)^{c-b-a}}{\sin (\pi c) \Gamma(a) \Gamma(b) \Gamma(1-c) \Gamma(2-c)} \sum_{r=0}^{\infty} \frac{(1-a)_{v}(1-b)_{v}}{\nu !(2-c)_{r}} z^{\prime}$

und

$\dot{F}(a, b ; c ; z) \approx \frac{\pi \Gamma(a+b-c) z^{1-c}(1-z)^{c-b-a}}{\sin (\pi c) \Gamma(a) I(b) \Gamma(1-c)} \sum_{\nu=0}^{\infty} \frac{(1-a)_{v}(1-b)_{\nu}}{v !(c-b-a+1)_{v}}(1-z)^{\nu}$.

Für $|z|>\sin \Phi_{\mid \zeta, 1}^{*} / \sin \left(|\zeta|+\Phi_{\mid \zeta i}^{*}\right)$ besitat $F$ in jedem der zuei Fälle

(vii) $\quad x>1 / 2, y \geqq 0,-\pi / 2-\Phi_{:}^{(1)}+\varepsilon \leqq \gamma \leqq-\pi / 2-\Phi_{i}^{*}+\varepsilon$,

(viii) $\quad \dot{x}>1 / 2, y<0, \pi / 2+\Phi_{|k|}^{*}-\varepsilon \leqq \dot{\gamma} \leqq \pi / 2-\Phi_{i}^{(-1)}-\varepsilon$

wiederum die asymptolische Enitwicklung (7).

Die Aussage des Satzes läßt sich im Fall $x>1 / 2, y \geqq 0$ in den Abbildungen (a), (b) veranschalulichen, wobei zur.Vereinfachung angenommen wird, daß $c$ auf einem vom Nullpunkt aus. gehenden Strahl gegen unendlich strebt. Die eingetragenen asymptotischen Entwicklungen gelten in den horizontal schraffierten (offenen) Winkelräumen unter den Voraussetzungen (4), $(\overline{5}),(6)$, in den vertikal schraffierten unter den Voraussetzungen (4), (5), (8). Durch Spicgelung an der reellen Achse der $c$-Ebene erhält man die entsprechenden Konfigurationen im Fall $y<0$.

Béispiele: Für $\zeta=\pi / 4$ berechnet man für $\Phi_{\zeta}^{*}$ einen Näherungswert von 1,5075 oder $86,37^{\circ}$. Daraus erhält man für $\sin \Phi_{\zeta}^{*} / \sin \left(\zeta+\Phi_{\zeta}^{*}\right)$ den Näherungswert 1,330 . Ist $z=3(1+i) / 4$, so ist wegen $|z| \approx 1,06$ nach $(2) \delta_{z} \equiv \Phi_{z}{ }^{(1)} \approx 1,5141 \triangleq 86,75^{\circ}$. Weiterhin ist $\Phi_{z}{ }^{(0)} \approx-1,3113$ $\triangleq-75,13^{\circ}$. Ist $z=10(1+\mathrm{i})$, also $|z| \approx 14,14$, so gilt nach $(2) \delta_{z}=\Phi_{*}^{*}$, und es ist $\Phi_{z}(0)$. $\approx-0,8115 \triangleq-46,49^{\circ}, \Phi_{z}^{(1)} \approx 1,5628 \triangleq 89,54^{\circ}$. Die in den Abbildungen (a), (b) vertikal schraffierten Winkelräume in der unteren $c$-Halbebene besitzen bei diesen Beispielen-relativ kleine Offnungswinkel. Aus Gleichung (2) ist erșichtlich, daß diese Aussage für alle Werte von $\zeta$, die nicht $z$ nahe bei Null liegen, aufechterhalten werden hann (s. dazu auch die nach. folgende Bemerkung 4).

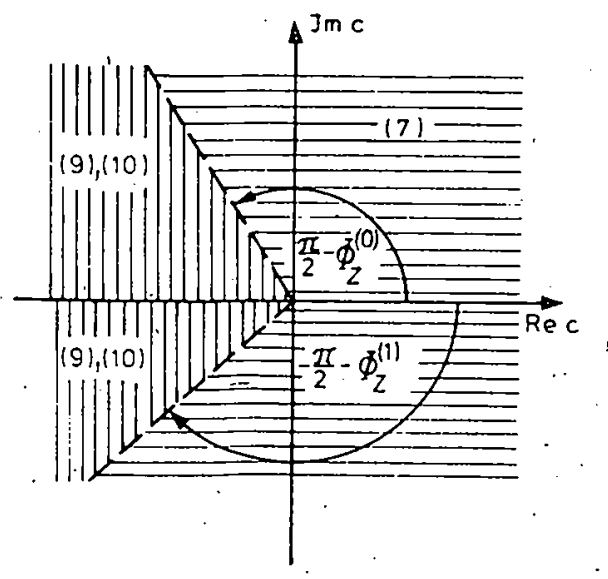

(a) $|z| \leqq \sin \Phi_{\zeta}^{*} / \sin \left(\zeta+\Phi_{?}^{*}\right)$

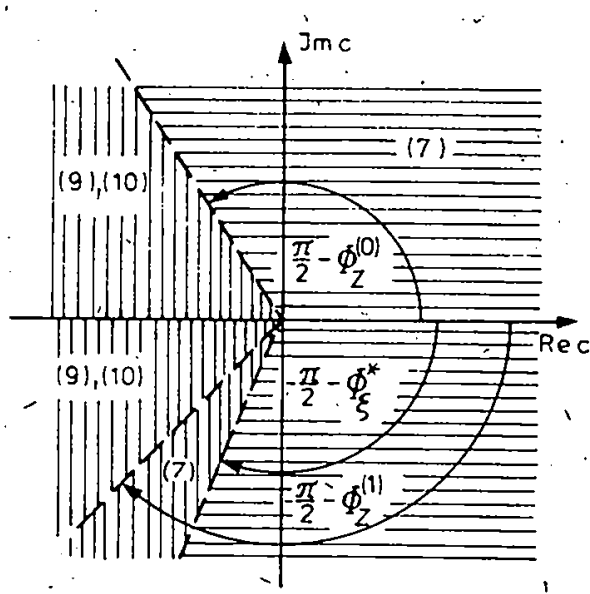

(b) $|z|>\sin \Phi_{\zeta}^{*} / \sin \left(\zeta+\Phi_{\zeta}^{*}\right)$

23 Antilysis Hd. 9, Heft 4 (1900) 
Bemerkungen: 1. Im Unterschied zu (9) tritt in der Entwicklung (10) nur noch ein Quotient zweier von c abhängiger Gammafunktionen auf. (Für diesen Quotienten gilt unter den Voraussetzungen (5) ebenfalls die in [9] zitierte asymptotische Entwicklung von TricomiErdélyi nach Potenzen von $1 / c$ mit polynomialen Koeffizienten in $a, b, z$ [10].) 2. Aus Beweispunkt 3.3 (s. (18)) wird ersichtlich werden, daß für $|z|>\sin \Phi_{\zeta}^{*} / \sin \left(\zeta+-\Phi_{\zeta}^{*}\right)$ die Beziehungen $\Phi_{\zeta}{ }^{*}<\Phi_{z}^{(1)}(y \geqq 0)$ und $\Phi_{|\zeta|}^{*}<-\Phi_{z}^{(-1)}(y<0)$ gelten, se daß die unter (vii) und (viii) angegebenen Winkelräume bei hinreichend kleiner Wahl von $\varepsilon>0$ nicht leer sind. 3. Der Winkel $\Phi_{\zeta}^{*}$ tritt in [9] nicht auf. Seine Einführung ist in der vorliegenden Arbeit beweistechnisch bedingt. Es ist zu' vermuten, daß er durch eine andere Beweisführung vermieden werden kann und sich dìnn die Fälle'(iii), (iv), (vii) und (viii) zu der Aussage_zusammenfassen lassen, daß unter den Voraussetzungen (4)-(6) die asymptotische Entwicklüng (7) in den Winkelräumen $-\pi / 2-\Phi_{z}{ }^{(1)}+\varepsilon \leqq \gamma \leqq \pi / 2-\Phi_{z}{ }^{(0)}-\varepsilon$ (für $x>1 / 2, y \geqq 0$ ) und $-\pi / 2-\Phi_{z}{ }^{(0)}+\varepsilon \leqq \dot{\gamma}$ $\leqq \pi / 2-\phi_{i}(-1)-\varepsilon$ (für $x>1 / 2, y<0$ ) gilt. 4. Für $\zeta \rightarrow+0$ ist auch $\Phi_{\zeta}^{*} \rightarrow+0$, so da $B$ man für $\Phi_{\zeta}^{*}$ die verkürzte Gleichung $\ln \Phi_{\zeta}^{*}-\ln \zeta+o(1)-(\pi+o(1)) / \Phi_{\zeta}^{*}=0$ erhält, alus dèr $\Phi_{\zeta}{ }^{*} \sim-\pi / \ln \zeta\left(\zeta-\zeta^{*}+0\right)$ folgt. Ferner, für. $\zeta \rightarrow \pi / 2-0$ ist auch $\Phi_{\zeta}^{*} \rightarrow \pi / 2-{ }^{\prime} 0$. Setzt $\operatorname{man} \zeta=\pi / 2-\bar{\zeta}$ und $\phi_{\zeta}^{*}=\pi / 2-\tilde{\Phi}_{\zeta}$, so gilt $\cos \tilde{\Phi}_{\zeta}=\cos \bar{\zeta} \exp \left\{\left(2 \pi-\bar{\zeta}-\tilde{\Phi}_{i}\right) \tan \tilde{\Phi}_{\zeta}\right\}$ und daraus erhält man für $\bar{\zeta} \rightarrow+0, \tilde{\Phi}_{\zeta} \stackrel{\because}{=}+0$ die verkürzte Gleichung $1+O\left(\tilde{\Phi}_{\zeta}^{2}\right)=1-\tilde{\zeta}^{2} / 2$ $+\left(2 \pi-\tilde{\zeta}_{)} \tilde{\Phi}_{i}+O\left(\tilde{\zeta}^{4}\right)+O\left(\tilde{\Phi}_{\zeta}{ }^{2}\right)+O\left(\tilde{\Phi}_{i} \tilde{\zeta}^{2}\right)\right.$. Aus ihr ergibt sich $\tilde{\Phi}_{\zeta} \sim \tilde{\zeta}^{2} /(4 \pi)$, und mithin ist. $\Phi_{6}^{*}-\pi / 2 \sim-(\zeta-\pi / 2)^{2} /(4 \pi)(\zeta \rightarrow \pi / 2-0)$.

\section{Beweis des Satzes}

3.1. Ohne Einschränkung der Allgemeinheit kann

$$
a=O(b), \quad b \rightarrow \infty
$$

vorausgesetzt werdén, denn $F$ ist symmetrisch in $a$ und $b$, und für konstante $a, b$ sind die Behauptungen des Satzes in [9] bewiesen.

3.2. Ausgangspunkt, fïr den Beweis in den Fällen (ii) - (viii) ist die bekannte Integraldarstellung

$F(a, b ; c ; z)=\frac{\Gamma(c)}{\Gamma(b) \Gamma(c-b)} \int_{0}^{1} t^{b-1}(1-t)^{c-b-1}(1-t z)^{-a} d t \cdot\left(c_{1}>b_{1}>0\right)$.

Wegen $(4)$ ist $(1-t z)^{-a}$ eine im Integrationsintervall beliebig oft differenzierbare Funktion von $t$, so da $\beta$ man mit der Taylorschen Formel

erhält, wobei

$$
F-\sum_{\nu=0}^{n} \frac{(a)_{\nu}(b)_{v}}{\nu !(c)_{\nu}} z^{\prime}=-\frac{\Gamma(c)}{\Gamma(b) \cdot \Gamma(c-b)} \frac{(a)_{n+1} z^{n+1}}{n !} \int_{0}^{1}(1-\grave{s})^{n} \Psi_{n}(a, b, c, s z) d \dot{s}
$$

$$
\psi_{n}^{\prime}=\int_{0}^{1} t^{b+n}(1-t)^{c-b-1}(1-s z t)^{-a-n-1} d t
$$

ist und wegen $(6) \cdot n$ so gro $B$ angenommen werden kann, da $B^{\prime}$

$$
\therefore b_{1}+n \geqq 0
$$

gilt. Durch dic Substitution $t=1-\mathrm{e}^{-r}$ erhält man die Darstellung von $\Psi_{n}$ als Laplace-Integral,

$$
\psi_{n}^{\prime}=\int_{0}^{\infty} \mathrm{e}^{-{ }^{-c \tau}} \psi^{\prime \prime}(a, b, \overline{s z}, \tau) d \tau
$$

mit der Originalfunktion $\psi_{n}=\mathrm{e}^{b z}\left(1-\mathrm{e}^{-r}\right)^{b+n}\left(1-s z+s z \mathrm{e}^{-r}\right)^{-z-n-1}$ 
Im nächsten Bẹweispunkt 3.3 werden wir zeigen, da $B \psi_{n}$ für jedes feste $z$ und alle $s \in[0,1]$ holomorphe Funk'tionen, von $\tau$ in den Winkelräumen

$$
W_{z}=\left\{\begin{array}{ll}
\{\tau \in \mathbb{C}:|\operatorname{Arg} \tau|<\pi / 2\} & \text { für } x \leqq 1 / 2, \\
\left\{\tau^{\prime} \in \mathbb{C}: \Phi_{z}{ }^{(0)}<\operatorname{Arg} \tau<\delta_{z}\right\} & \text { fuir } x>1 / 2, y \geqq 0, \\
\left\{\tau \in \mathbb{C}:-\delta_{z}<\operatorname{Arg} \tau<\Phi_{z}{ }^{(0)}\right\} & \text { fuir } x>1 / 2, y<0
\end{array} .\right.
$$

sind. $\mathrm{Da}$ in ' $W_{\text {: auBerdem }} \psi_{n}=O(1)$ für' $\tau \rightarrow 0$ und $\psi_{n}=O\left(\mathrm{e}^{\left|\dot{b}_{\mathrm{r}}\right|}\right)$ für $\tau \rightarrow \infty$ gilt, könnẹ nach einem bekanntén Satz [3: Kap. IX, $\S 2$ ] die Funktionen $\Psi_{n}$ bezüglich $c$ analytisch fortgesetzt werdèn, indem man in (13) den Integrationsweg innerhalb

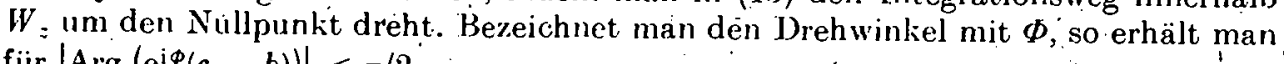
fiir $\left|\operatorname{Arg}\left(\mathrm{e}^{\mathrm{i} \Phi}(c-b)\right)\right|<\pi / 2$

$$
\left.F-\sum_{\nu=0}^{n} \frac{(a)_{v}(b)_{v}}{\nu !(c)_{v}} z^{\nu}=\frac{\Gamma(c)(a)_{n+1} z^{n+1}}{\Gamma(b) \Gamma(c-b) n !} \int_{0}^{1}(1-s)^{n} \int_{0}^{\infty} \mathrm{e}^{-c \tau} \psi_{n} d \tau d s,{ }^{1}\right)
$$

also eine Darstellung von $F$, die wegen $b=\delta(c)$ fïr hinreichend große $|c|$ und

$$
|\gamma+\Phi| \leqq \pi / 2-\varepsilon
$$

gilt. Damit werden die in den Fällen (ii) - (iv) angegebeneñ Winkèlräume erfa ß̣t.

3.3. Die Holomorphie von $\psi_{n}$ in $W_{z}$ folgt aus der Nullstellenfreiheit von $1 .-\mathrm{e}^{-}$. und $1-s z+s z \mathrm{e}^{-\mathrm{r}}$ in $W_{z}$, so dạ

$$
-\log (1-1 / s z)+2 k r \dot{\mathrm{i}} \notin W_{z} \quad(s \in(0,1] ; k=-1,0,1) \text {, }
$$

nachzuweisen ist. Für Re $(s z)=s x \leqq 1 / 2$ ist. $\left|1^{\prime}-1 / s z\right| \geqq 1$ und deprnach (16) in dèn beiden Fällen $x \leqq 1 / 2,0<s \leqq 1$ und $x>1 / 2,0<s \leqq 1 / 2 x$ giiltig. Auch für $1 / 2<z=x<1,1 / 2 x^{\prime} \leqq s \leqq 1$ ist (16) leicht nachprufbar. Wir betrachten nun den Fall $x>1 / 2, y>0,1 / 2 x \leqq s \leqq 1$. Dabei laufen für jedes feste $z$ die Punkte $1-1 / s z$ $=\varrho_{i}(s) \exp \left[i \vartheta_{z}(s)\right]$ mit wachsenden $s$ auf der Geraden $g_{z}=\sin \zeta / \sin \left(\zeta+\vartheta_{z}\right)$ vom Punkt $\vartheta_{z}=\pi-2 \zeta, o_{z}=1$ in das Innere des Einheítskreises, und $\vartheta_{z}(s)$ fällt echt monoton. Also ist

mit.

$$
\operatorname{Arg}(-\log (1-1 / s z))=\arctan f\left(\vartheta_{z}\right)
$$

$$
f\left(\vartheta_{z}\right)=\vartheta_{z} / \ln \left(\sin \zeta / \sin \left(\zeta+\vartheta_{z}\right)\right):\left(0<\operatorname{Arg}(1-1 / z) \leqq \vartheta_{z}<\pi-2 \zeta\right)
$$

Die Funktion $f$ fällt echt monoton mit wachsenden $\vartheta_{z}$, so da: $\beta$

$$
\operatorname{Arg}(-\operatorname{Iog}(1-1 / s z)) \leqq \Phi_{i}{ }^{(0)}
$$

gilt. Andererseits ist, wénn man $\vartheta_{z}=x \perp \zeta^{\circ}-\Phi_{z}$ setzt,

mit

$$
\operatorname{Arg}(-\log (1-1 / s z)+2 \pi i)=\arctan f\left(\Phi_{z}\right)
$$

$$
f\left(\Phi_{z}\right)=\left(\pi+\zeta+\Phi_{z}\right) / \ln \left(\sin \Phi_{z} / \sin \zeta\right) \quad\left(\zeta<\Phi_{z}<\pi-\zeta\right)
$$

Wegen $f^{\prime}(\Phi)=g_{r}(\Phi) \ln ^{2}[\sin \Phi / \sin \zeta]$ (s. (1)) besitzt $f$ ein eindeutig bestimmtes Minimum in $\Phi=\Phi_{?}^{*}$. Ist $|z| \geqq \sin \Phi_{?}^{*} / \sin \left(\zeta+. \Phi_{?}^{*}\right)$ oder die dazu äquivalente Ungleichung $\cot \Phi_{\urcorner}{ }^{*} \geqq(1-x) / y=\cot (x-\operatorname{Arg}(z-1))$ erfiillt, so folgt die Un-

1) Im zweiten Integral in (14) bedeutet $\omega(\Phi)$, daB über cinen vom Nullpunkt ausgehenden Strahl, der den Winkel $\$$ mit der rcellen Achse der $\tau$-Ebene cinschließt, integriert wird. 
gleichung. $\Phi_{\xi}^{*} \leqq \pi,-\operatorname{Arg}(z-1)=\pi-\zeta-\vartheta_{i}(1)=\Phi_{i}(1)$, (ind, demnach ist

$$
\operatorname{Arg}(-\operatorname{Iog}(1-\cdot 1 / s z)+2 \pi i) \geqq \arctan f\left(\Phi_{:}^{*}\right)=\Phi_{;}^{*}=\delta_{z} \cdot
$$

Offenbar erhält man aus $|z| \leqq \sin \Phi_{:^{*}}^{*} / \sin \left(\zeta+\Phi_{\imath^{*}}^{*}\right)$ auf dieselbe Weise $\Phi_{:}^{*} \geqq \Phi_{z}(1)$, und damit

$\operatorname{Arg}(-\log (1-1 / s z)+2 \pi \mathrm{i}) \geqq \operatorname{Arg}(-\log (1-1 / z)+2 \pi \mathrm{i})=, \Phi_{z}^{(1)}=\delta_{z} \cdot$

Aus (17)-(19) folgt (16).

Für $z$ mit $x>1 / 2, y<0$ erhält man (16) nach Übergang $z u, \bar{z}$ bei Beachtung der Relationen Arg (-Log $(1-1 / s z)+2 k \pi \mathrm{i})=-\operatorname{Arg}(-\operatorname{Jog}(1-1 / s \bar{z})-2 k \pi \mathrm{i})$ ebenfalls aus $(17)-(19)$.

3.4. Die Entwicklung (7) folgt aus (14), wenn für jede hinreichènd große natürliche Zahl $n$ und glẹchmäßig fiir alle $s \epsilon^{\prime}[0,1]$

$$
\int_{0}^{\infty(\phi)} \mathrm{e}^{-c r} \psi_{n} d \tau \sim \Gamma(b+n+1) / c^{b+n+1} \quad(|c| \rightarrow \infty ;|\gamma+\Phi|<\pi / 2-\varepsilon)
$$

gilt, so daß das Restglied die Ordnung des 'ersten' vernachlässigten Gliedes hat, denn nach der Stirlingschen, Formel ist.

$$
\frac{\ddot{\Gamma}(c)}{\Gamma(c-b) c^{b+n+1}} \sim c^{-n-1} \sim 1 /(c)_{n+1} . \quad(|c| \rightarrow \infty ;|\gamma| \leqq i-\varepsilon)
$$

Zum Beweis von (20) deformieren' wir den Integrationsweg in einer kleinen Um-gebung des Nullpunktes. Dabei verwenden wir die folgenden Bezeichnungen (in Anlehnung an [2]):

$$
\tilde{b}=b+n, \quad X=\vec{b} / \dot{c}, \quad \omega=\sqrt[4]{|\bar{b}|} x / \sqrt{\bar{b}}, \quad \delta=\left|\vec{b}^{5 / 4} / c\right| \ln |c / b| \vdots
$$

$r$ sei diejenige positive Zahl, für die $|X+r \omega|=\delta$ ist. Der deformierte Integrationsweg soll aus dem die Punkte $0, X-\omega, X+\omega$ und $X+\dot{r} \omega$ in der angegebenen Reihenfolge verbindenden Polygonzug bestehen und auf einem Kreisbogen $|\tau|=\delta$ von $X+r \omega$ zum ursprünglichen Integrationsweg zurïckführen. I)ie Integrale iaber die cinzelnen Wegstiicke bezeichnen wir in der Reihenfolge ihrer Durchlaufung mit $I_{1}$ bis $I_{5}$. Im folgenden Beweispunkt zeigen wir.

$$
I_{2}=\int_{x-\omega}^{x+\dot{\omega}} \mathrm{e}^{-c \mathrm{r}} \psi_{n} d \tau \sim \Gamma(b+n+1) / c^{b+\eta+1} \quad(|c| \rightarrow \infty)
$$

und daran anschließend in den Beweispunkten 3.6-3.9 $I_{\nu}=o\left(I_{2}\right)(v=1,3,4,5)$ für $|c| \rightarrow \infty,|\gamma+\Phi| \leqq \pi / 2-\varepsilon$. Zu diesem $/ / w e c k$ vermerken wir noch die folgenden wichtigen Beziehungen, die aus (5) und (11) folgen:

$$
\omega=o(X), \quad \dot{X}=o(\delta), \quad \delta \stackrel{\prime}{=} o(1) ; \quad|r \omega| \sim \delta .
$$

3.5. Setzt man $k(\tau)==^{\prime} c \tau-\tilde{b} \log \tau(\log \tau>0$ fiir $\tau>1)$ und $H(\tau)=e^{b_{2}}\left[\left(1-e^{-\tau}\right) / \tau\right]^{\bar{b}}$ $\times\left(1-s z+s z \mathrm{e}^{-\tau}\right)^{-a-n-1}$, so kann $I_{2}$ in der Form $I_{2}=\int_{x-\omega}^{x+\omega} \mathrm{e}^{-k} H d \tau$ geschrieben werden. Dann ist mit (21) $k(X)=\tilde{b}-\tilde{b} \log (\bar{b} / c), \quad k^{\prime}(X)=0 ; \omega^{2} k^{\prime \prime}(X)=\sqrt{|\bar{b}|} \rightarrow \infty$. Man sieht leicht, daß gleichmäßig für alle $s \in[0,1]$

$$
H \sim 1 \quad \text { für } \tau \doteq o(1 / b) .
$$


gilt. Wegen $X+\vartheta \omega \sim X$ für alle $\vartheta \in[-1,1]$ und $X \doteq{ }_{,} o(1 / b)$ gilt iinsbesondere $H(X+\vartheta \omega) \sim 1$ gleichmäßig für alle $s \in[0,1]$. Damit ist gezeigt, da $B$ die Voraussetzungen des Satzes 20.3 aus [2] erfüllt sind, und man erhält mit ihm

$$
I_{2} \sim \sqrt{2 \pi \bar{b}} \mathrm{e}^{-\tilde{b}} X^{\tilde{\delta}} / c \quad(c \rightarrow \infty) .
$$

Diese asymptotische - J)arstellung ist wegen $\Gamma(b \mp n+1) \sim \sqrt{2 \pi b} b^{n+b} \mathrm{e}^{-b}$ und $X^{\bar{b}} \sim \mathrm{e}^{u}(b / c)^{b \div n}$ äquivalent $\mathrm{zu}(22)$.

3.6. Fiir das Jntegral $I_{1}$ von 0 nach $X-$ - erhält man wegen (24)

$$
\left|I_{1}\right| \leqq 2\left|(X-\omega)^{\tilde{b}+1}\right| \int_{0}^{1} \mathrm{e}^{k(u)} d u
$$

mit den Bezeichnungen $k(u)=-\operatorname{Re}[c(X-\omega)] u+\tilde{b}_{1} \ln u=-\left[\tilde{b}_{1}-|\bar{b}|^{3 / 4}\right.$ $\times \cos (\bar{\beta} / 2)] u+\bar{b}_{1} \ln u, \tilde{b}_{1}=b_{1}+n, \tilde{\beta}=\operatorname{Arg} \tilde{b} \in[-\pi / 2, \pi / 2]$. Da $k^{\prime}$ in $(0,1)$ monoton fällt und $k^{\prime}(1)=\left|b^{3 / 4}\right| \cos (\tilde{\beta} / 2)$ positir ist, gilt

$$
\int_{0}^{1} \mathrm{e}^{k(u)} d u \leqq \mathrm{e}^{k(1) / k^{\prime}(1)}
$$

Beruicksichtigt man noch $-(X-\omega)^{\tilde{b}+1}=X^{\bar{b}+1} \exp \{-\sqrt[4]{|\bar{b}|} \sqrt{\tilde{b}}-\sqrt{|\tilde{b}|} / 2+O(\sqrt[4]{\tilde{b}})\}$ so erhält ma! mit (11), (23), (25) aus (26), (2) die Beziehung

$$
\left|I_{1} / I_{2}\right| \leqq \exp \{-\sqrt{|\tilde{b}|} / 4\}=o(i) \text {. }
$$

3.7. Es ist

$$
I_{3}=\int_{x+\omega}^{x+r \omega} \mathrm{e}^{-(\varepsilon-0) \tau}\left(1-\mathrm{e}^{-\tau}\right)^{\tilde{b}}\left(1-s z+s z \mathrm{e}^{-\tau}\right)^{-a-n-1} d \tau .
$$

Nach (23)-gilt a.if dem (gérad̀inigen) Integrations̀weg $\tau=O(\delta)=o(1)$ und folglich $\left(1-\mathrm{e}^{-\tau}\right)^{\tilde{b}}=\tau^{\tilde{b}} \mathrm{e}^{\dot{0}(\delta \delta)},\left(1-s z+s z \mathrm{e}^{-\tau}\right)^{-a-n-1}=\mathrm{e}^{o(b \delta)}$. Damit erhält man die Abschätzung

$$
\left|I_{3}\right| \leqq 2\left|X^{\tilde{b}} b^{3 / 4} / c\right| \mathrm{e}^{-\tilde{b}+O(b \delta)} \int_{1}^{r} \mathrm{e}^{\mathrm{Re} k(u)} d u
$$

mit $k(u)=\stackrel{\prime}{-}(c-b) \omega u+\tilde{b} \log (1+u \omega / X)$. Wegen.

$$
\operatorname{Re} k^{\prime \prime}(u)=-\left|\sqrt{\bar{b}} X^{4} /(X+u(u))^{4}\right| \operatorname{Re}\left\{\left(1+u \cdot \sqrt{\bar{b}} /|\bar{b}|^{3 / 4}\right)^{2}\right\}<0
$$

ist $\operatorname{Re} k^{\prime}(u) \leqq \operatorname{Re} k^{\prime}(1) \sim-\sqrt{|\bar{b}|}$ und demnach

$$
\left.\int_{1}^{r} \mathrm{e}^{\operatorname{Re} k(u)} d_{1}\right\rfloor \leqq \mathrm{e}^{\operatorname{Re} k(1)} /-\operatorname{Re} k^{\prime}(1)=\mathrm{e}^{-\sqrt{\bar{b}} / 2+\dot{O}\left(b^{1 / \alpha}\right)}: .
$$

Aus (28) und (29) errechnet man mit (25) dic Bezichıng

$$
\left|I_{3} / I_{2}\right| \leqq \exp \left\{-\sqrt{\tilde{b}} / 2+O\left(b^{1 / 4}\right)+O(b \delta)\right\}=o(1) .
$$


3.8. Auf dem Kreisbogen' mit dem Mittelpunkt 0 und,dem Radius $\delta$ rom Punkt $X+\dot{r} \omega$ nach $\delta \mathrm{e}^{\mathrm{i} \Phi}$ ist wegen $|\tau|=\delta=o(1)$

$$
\left|\left(1-\mathrm{e}^{-\tau}\right)^{\tilde{\delta}}\right| \leqq\left|\delta^{\dot{\delta}}\right| \mathrm{e}^{O(b)}, \quad\left|\left(1-s z+s z \mathrm{e}^{-\tau}\right)^{-a-n-1}\right| \leqq \mathrm{e}^{O(b \delta)} .
$$

Folglich gilt

$$
\left|I_{4}\right| \leqq\left|\delta^{\tilde{b}+1}\right| \mathrm{e}^{O(b)} \int_{n_{0}+\gamma}^{\phi+\gamma} \mathrm{e}^{-\delta ! c i \cos u(1+\delta(1))}\left|d u u^{\prime}\right|
$$

mit $u_{0}=\operatorname{Arg}(X+r \omega) \sim \operatorname{Arg}(r \omega)=\operatorname{Arg}(1)=\bar{\beta} / 2-\gamma$. Es ist unmittelbarklar, da $\beta$ wegen (15) und $\hat{\beta} \in[-\pi / 2 ; \pi / 2]$ das Integiationsintervall in $[-\pi / 2+\varepsilon, \pi / 2-\varepsilon]$ liegt, also $\cos u \geqq \sin \varepsilon$ und damit

$$
\left|I_{s}\right| \leqq\left|\delta^{\tilde{b}+1}\right| \mathrm{e}^{-\delta: c \cdot \sin \varepsilon(1: \theta(1))}
$$

gilt. Aus (25) und (30) erhält man die Beziehung

$$
\left|I_{4} / I_{2}\right| \leqq \exp \left\{-2^{-1}\left|b^{5 / 4} \ln \right| c / b \mid \sin \varepsilon\right\}=o(1) .
$$

3.9. Auf dem Strahl mit der Parameterdarstellung $\tau=(\delta+u) \mathrm{e}^{\mathrm{i} \Phi}(0 \leqq u<\infty)$ gelten wegen $\mid \Phi !<\pi / 2$ und (11) die Ungleichungen $\left|\left(1^{i}-\mathrm{e}^{\cdot r}\right)^{\tilde{b}}\right| \leqq \mathrm{e}^{O(b)}, \mid(1-\delta z$ $\left.+s \dot{z} \mathrm{e}^{-r}\right)^{-a-n-1} \mid \leqq \mathrm{e}^{O(b)}$. Daraus folgt wegen (15) $\left|I_{5}\right| \leqq O_{1}\left(|c|^{-1} \exp \left\{-2^{-1} \delta|c| \sin \varepsilon\right.\right.$ $+O(b)\})$ und mit (25)

$$
\left|I_{5} / I_{2}\right| \leqq \exp \left\{-2^{-1} \delta|c| \sin \varepsilon(1+o(1))\right\}=o(1) .
$$

Damit ist der Beweis in den Fällen (ii) - (iv) erbracht.

3.10. Die Beweismethode in den rèstlichen Fällen (v)-(viii) ist prinzipiell dieselbe wie in [9], so daß sie hier nur liurz skizziert wird. Ausgangspunkt ist die bekannte Beziehung

$$
\begin{aligned}
F(a ; \dot{b} ; c ; z)= & k_{1} F(u, \dot{b} ; \dot{a}+b-c+1 ; 1-z) \\
\vdots & +k_{2} F(1-a, 1-b ; 2-c ; z)
\end{aligned}
$$

mit den Koeffizienten

$$
\begin{aligned}
& k_{1}=\frac{\Gamma(a-c+1) \Gamma(b-c+1)}{\Gamma(a+b-c+1) \Gamma(1-c)} \\
& k_{2}=\frac{\pi z^{1-c}(1-z)^{c-b-a} \Gamma(a-c+1) \Gamma(b-c+1)}{\sin (\pi c) \Gamma(a) \Gamma(b) \Gamma(1-c) \Gamma(2-c)} .
\end{aligned}
$$

Nach der Stirlingschen Formel gelten in den unter (v)-'(viii) angegebenen Winkel- . - räumen unter den Voraussetzungen (8) und (11)

$$
k_{1} \sim 1, \quad k_{2}=\frac{z^{-c}(1-z)^{c}}{\sin (\pi c)} \exp \{O(b \log (-c))\} \quad(c \rightarrow \infty)
$$

Die hypergeometrischen Funktionen auf der rechten Seite von (31) können únter der Voraussetzung (8) für alle $c$ mit $c_{1} \leqq 0$ gemäB (7) in asymptotische Reihen entwickelt werden. Deshalb besitzt $F(a, b ; c ; z)$ dieselbe asymptotische Entwicklung wie 
der erste Summand in (31); falls $k_{2}$ für $c \rightarrow \infty$ stärker als jede Potenz von $1 / c$ gegen null strebt. Andererseits besitzt $F(a, b ; c ; z)$ dieselbe Entwicklung wie der zweite Summand in (31), falls $k_{2}$ für $c \rightarrow \infty$ stärker als jede Potenz von $c$ wächst. Man überlegt sich leicht, da $B$ in den unter (v) und (vi) angegebenen Winkelräumen die ziveite Variante zutrifft, womit (9) bewiesen ist. Dagegen gilt für $x>1 / 2$ in den Winkelräumen

$$
\begin{aligned}
& -\pi / 2-\Phi_{z}{ }^{(1)}+\varepsilon \leqq \gamma \leqq-\pi / 2, \quad \pi / 2 \leqq \gamma \leqq \pi / 2-\Phi_{z}(0)-\varepsilon \quad(y \geqq 0) ; \\
& \because \pi / 2-\Phi_{z}{ }^{(0)}+\varepsilon \leqq \gamma \leqq-\pi / 2, \quad \pi / 2 \leqq \gamma \leqq \pi / 2-\Phi_{z}{ }^{(-1)}-\varepsilon \quad(y<0)
\end{aligned}
$$

die, asymptotische Entwicklung

$$
F(a, b ; c ; z) \approx k_{1} \sum_{\nu=0}^{\infty} \frac{(a)_{r}(b)_{\nu}}{\nu !(a+b-c+1)_{r}}(1-z)^{\prime}
$$

Da sich $k_{1}$, wie aus [10] hervorgeht, unter den Voraussetzungen (5) ebenfalls in eine asymptotische Reihe nach fallenden Potenzen von $c$ entwickeln läßt, deren-Koeffizienten Polynome'in $a, b$ sind, ist auch die rechte Seite von (32) in eine solche Reihe entwickelbar. Aus dieser kann' man 'eine asymptotische Entwicklung der Form . $\Sigma P_{r}(a, b, z) /(c)_{v}$ erhalten, bei der die $P_{v}$ Polynome in $a, b, z$ sind. Wegen der Eindeutigkeit asymptotischer Entwicklungén folgt durch Koeffizientenvergleich in den Winkelräumen ${ }^{\prime} \pi / 2 \leqq|\gamma| \leqq \pi / 2+\varepsilon$, da $\beta P_{v}=(a),(b)_{v} z^{\prime} / v$ ! ist. Daraus folgt die Behauptung in den Fällen (vii) und (viii). Ersetzt man noch in der eben bewiesenen Entwicklung

$$
\frac{\Gamma(a-c+1) \Gamma(b-c+1)}{\Gamma^{\prime}(a+b-c+1) \Gamma(1-c)} \dot{\vdots}(a, b ; a+b-c+1 ; z) \approx \sum_{\nu=0}^{\infty} \frac{(\alpha)_{\nu}(b)_{v}}{\nu !(c)_{v}} z^{\gamma}
$$

die Gıößen $a, b, c, z^{\bullet}$ in dieser Reihenfolge durch $1-a, 1-b, c-b-a+1$ und $1-z$, so erhält man aus (9) die Entwicklung (10)

\section{LITERATUR}

[1] Batemax, H., and A. Endélyi: Higher 'Transcendental Functions, Vol. 1. New-YorkToronto-London: Mc'Graw.Hill Book Company, Inc. 1953.

[2] BerG, L.: Asymptotische Darstellungen und Entwicklungen. Berlin: Dt. Verlag Wiss. 1968.

[3] Doztscir, G:: Handbuch der Laplace-Transformation I (Nachdruck der 1. Auflage). Basel-Stuttgart: Birkhäiser Verlag 1972.

[4] Хапаев, М. М.: Асимптотические разложения гипергеометрических и вырондё̈ных гиергеометрических фуінций. Изљ. высыл. уч. зањ. Мат. 24 (1961) 5, 98-101.

[5] Perrox; O.: Uber diss Verhalten der hypergeometrischen Reihe bei unbegrenztem Wachstum eines oder mehrerer Parameter I, I I Sitzungsber. Heidelberger Akad. Wiss. 7 (1916) $9,1-24$, und 8 (1917) 1, 1-69.

[6] Wagnes, E.: Asymptotische Entwicklungen der hypergeometrischen Funktionen $F(a, b, c ; z)$ für $|\bar{a}| \rightarrow \infty$ und konstante $b, c, z$. Z. Anal.; Anw. 3 (1984), 213-226.

[7] WaGner, E.: Asymptotische Darstellungen der hypergeometrischen Funktion' für große Parameter unterschiedlicher Größenordnung. Z. Anal. Anw. 5 (1986), 265-276.

[8] WaGier, E.: Zur Asymptotik der hypergeometrischen Funktionen für große Parameterwerte. Wiss. $Z$. Univ. Halle, Math.-Naturwiss. R. 34 (1985) 4, 38-42. 
[9] Wagser, E.: Asymptotische Entwicklungen der hypergeometrischen Funktion $F(a, b, c, z)$ für $|c| \rightarrow \infty$ und konstante Werte $a, b$ und $z$. Demonstratio Math. 21 (1988), 441-458.

[10] WaGser, E.: Asymptotische Entwicklung des Quotienten zweier Gammafunktionen. Wiss. Z. Univ. Halle (erscheint).

[11] Watsox, G. N.: Asymptotic expansions of hypergeometric functions. Trans. Cambridge Phil. Soc. 22 (1918), $277-308$.

Manuskripteingang: 05.05 .1989 ; in revidierter Fassung 09. 08.1989

\section{VERFASSER:}

Dr. Sc. EBfrhard WaGNer

Sektion Mathematik der Martin-Luther-Universität

Universitätsplatz 6

DDR - 4010 Halle 